\title{
ESTUDO DE ADSORÇÃO DE COMPOSTOS SULFURADOS UTILIZANDO UM DIESEL COMERCIAL DOPADO COM BENZOTIOFENO E DIBENZOTIOFENO
}

\author{
Robison P. Scherer, Álvaro L. Malvesti e Sibele B. C. Pergher* \\ Departamento de Química, Universidade Regional Integrada do Alto Uruguai e das Missões, Av. Sete de Setembro, 1621, \\ 99700-000 Erechim - RS, Brasil \\ Wladmir Ferraz de Souza \\ PETROBRAS/CENPES/THPE, Ilha do Fundão, Quadra 7, 21949-900 Rio de Janeiro - RJ, Brasil
}

Recebido em 29/10/07; aceito em 24/7/08; publicado na web em 20/1/09

\begin{abstract}
STUDY OF ADSORPTION OF SULPHUR COMPOUNDS EMPLOYING A COMERCIAL DIESEL DOPPED WITH BENZOTHIOPHENE AND DIBENZOTHIOPHENE. Desulphurization process by adsorption was studied employing a commercial diesel dooped with $1000 \mathrm{mg} / \mathrm{L}$ of benzothiophene and dibenzothiophene. The adsorbents materials employed were three types of activated alumina (acid, basic and neutral). For comparison, adsorption process was made also using oxidized diesel sample. The results showed that the adsorbents were selective for sulphur compounds removal from fuels. The contact time have influence in adsorption process achieving $80 \%$ of removal for not oxidized dibenzothiophene. The three studied alumina types showed similar behavior and a greater selective in dibenzothiophene adsorption than benzothiophene. Dibenzothiophene removal is more effective in samples not oxidized, whereas the benzothiophene was almost totally removed in oxidized sample.
\end{abstract}

Keywords: sulphur compounds; adsorption; fuels.

\section{INTRODUÇÃO}

Compostos contendo enxofre estão presentes nos derivados de petróleo em concentrações bastante significativas. A presença de compostos sulfurados em frações de petróleo é altamente indesejável, devido à ação corrosiva e poluição atmosférica promovida por gases prejudiciais ao meio ambiente gerados durante a combustão, tais como $\mathrm{SO}_{2}$ e $\mathrm{SO}_{3}$. Com isso, a exigência por combustíveis mais limpos e um rigoroso controle destes poluentes emitidos por automóveis tem levado a uma necessidade de maiores pesquisas na área de remoção de sulfurados em combustíveis, sobretudo diesel e gasolina.

Visando uma redução de compostos sulfurados, tem-se buscado constantemente o desenvolvimento de tecnologias para remoção de enxofre de combustíveis. Estudos recentes utilizando processo de adsorção empregando adsorventes seletivos têm se mostrado eficientes na remoção deste poluente. ${ }^{1-6}$

Nas refinarias, a remoção destes contaminantes de combustíveis é executada através do processo de hidrotratamento (hidrogenação catalítica) que também ajusta a especificação dos combustíveis, tal como teores de aromáticos e/ou hidrocarbonetos instáveis. Especificamente, a remoção de enxofre é feita através do processo de hidrodessulfurização (HDS). ${ }^{7}$

Outras rotas alternativas ao hidrotratamento têm sido também usadas com o objetivo de remoção destes contaminantes naturais de derivados de petróleo, tais como o oxitratamento de correntes de derivados instáveis através do uso de um sistema Fenton. ${ }^{8,9}$

Além disso, o oxitratamento de diesel pós-hidrotratado tem sido aplicado conjuntamente com uma etapa de adsorção posterior com alumina para remoção de sulfurados oxidados visando a remoção profunda dos sulfurados. ${ }^{10}$

O objetivo desse trabalho foi investigar o processo de dessulfurização por adsorção de um diesel comercial dopado com benzotiofeno

*e-mail: pergher@uri.com.br e dibenzotiofeno usando como material adsorvente três tipos de aluminas ativadas (ácida, básica e neutra). Escolheu-se trabalhar com o diesel comercial no lugar de solventes, como cicloexano, normalmente empregados com o propósito de empregar uma matriz similar aos óleos que são tratados na refinaria. A critério de comparação o processo de adsorção foi realizado também em amostras de diesel oxidado utilizando-se como agente oxidante o peróxido de hidrogênio $\left(\mathrm{H}_{2} \mathrm{O}_{2}\right)$ a $30 \%$ e ácido acético. ${ }^{11,12}$

\section{PARTE EXPERIMENTAL}

\section{Amostras}

A amostra em estudo é um diesel comercial dopado com os compostos sulfurados benzotiofeno (95\% da Sigma Aldrich) e dibenzotiofeno (98\% da Sigma Aldrich) (Figura 1).

A critério de comparação, também se empregou uma amostra de diesel oxidada, sendo esta dopada somente com o sulfurado dibenzotiofeno.

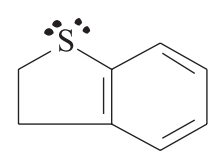

(a)<smiles>C1=CC2[Si]c3ccccc3C2C=C1</smiles>

(b)

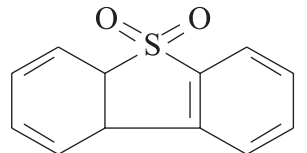

(c)
Figura 1. Compostos sulfurados: (a) benzotiofeno; (b) dibenzotiofeno e (c) dibenzotiofeno oxidado

\section{Adsorventes}

Empregaram-se como adsorventes três tipos de aluminas ativadas da Merck (granulometria 0,063-0200 mm) denominadas comercialmente de ácida, básica e neutra, devido possuírem estas características. 


\section{Adsorção}

O processo de adsorção foi realizado empregando $20 \mathrm{~mL}$ de amostra e $100 \mathrm{mg}$ de alumina. $\mathrm{O}$ procedimento foi realizado sob agitação $(125 \mathrm{rpm})$ e a temperatura de $40{ }^{\circ} \mathrm{C}$. O tempo de contato foi estudado: 1, 2, 3, 4, 12 e $24 \mathrm{~h}$.

\section{Cromatografia gasosa (GC/PFPD)}

As amostras antes e após o processo de adsorção foram analisadas via cromatográfica gasosa empregando um cromatógrafo CP - 3800 com detector PFPD. A Tabela 1 apresenta as condições utilizadas.

Tabela 1. Condições de análise para o GC/PFPD

\begin{tabular}{lc}
\hline Parâmetro & Condição \\
\hline Temperatura inicial & $50{ }^{\circ} \mathrm{C}$ \\
Tempo inicial & $2 \mathrm{~min}$ \\
Rampa de aquecimento & $8{ }^{\circ} \mathrm{C} / \mathrm{min}$ \\
Temperatura final & $300^{\circ} \mathrm{C}$ \\
Coluna & $\mathrm{CP}-\mathrm{Sil} 8 \mathrm{CB}(30 \mathrm{~m}$ x $0,32 \mathrm{~mm})$ \\
Gás de arraste (fluxo constante) & Nitrogênio \\
Modo de injeção & Split $(1: 10)$ \\
Velocidade linear do gás de & 2 mL/min \\
arraste & variável \\
Pressão da coluna & 43,25 min \\
Tempo total de corrida &
\end{tabular}

\section{Processo de oxidação}

Para a reação de oxidação preparou-se uma solução de concentração $1000 \mathrm{mg} / \mathrm{L}$, do composto sulfurado dibenzotiofeno/diesel comercial. Os experimentos foram realizados empregando-se 150 $\mathrm{mL}$ de diesel dopado a temperatura constante de $80^{\circ} \mathrm{C}$.

Os volumes dos reagentes utilizados no processo foram de $16,0 \mathrm{~mL}$ de peróxido de hidrogênio $\left(\mathrm{H}_{2} \mathrm{O}_{2} 30 \%\right)$ e 25,0 $\mathrm{mL}$ de ácido acético glacial. ${ }^{11,12}$

Após a oxidação, a fase oleosa sofreu duas lavagens com soluções de salmoura para eliminar os traços de oxidante ainda presentes na amostra.

\section{Caracterização dos materiais}

Os materiais empregados foram caracterizados por: difração de raios-X, análise textural por adsorção de nitrogênio e espectroscopia no infravermelho.

As análises de difração de raios $\mathrm{X}$ foram realizadas num Diffraktometer modelo D5000 (Siemens) utilizando filtro de Ni e radiação $\mathrm{Cu}-\mathrm{k} \alpha(\lambda=1,54 \AA$ ) $)$.

A caracterização textural dos materiais foi realizada utilizando um Autosorb-1 da Quantachrome (Nova-2200e). Antes da análise, cerca de $100 \mathrm{mg}$ de amostra foram tratadas a vácuo, a uma temperatura de $300{ }^{\circ} \mathrm{C}$ por $3 \mathrm{~h}$. As medidas foram realizadas na temperatura do $\mathrm{N}_{2}$ líquido.

A análise de espectrocopia no infravermelho de refletância difusa com transformada de Fourier (DRIFTS) foi realizada em um espectrofotômetro FTIR Shimadzu, modelo 8300. O espectro foi obtido com 32 varreduras e com $4 \mathrm{~cm}^{-1}$ de resolução.

\section{RESULTADOS E DISCUSSÃO}

\section{Caracterização dos adsorventes}

As aluminas empregadas foram caracterizadas por diversas técnicas complementares. A seguir apresentam-se estes resultados.

Através da análise de difração de raios - X (Figura 2) observouse que os materiais apresentaram um difratograma característico da alumina $\gamma$, a qual é um composto pouco cristalino, pois apresenta basicamente modulações de intensidades fracas e difusas. Esse padrão de difração é compatível com o modelo de rede aleatória, e tem sido atribuído à tendência de organização a curta distância.

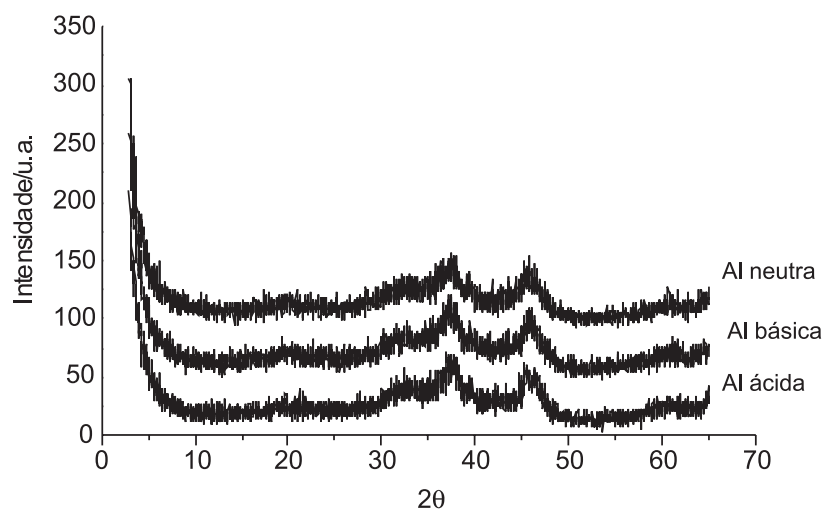

Figura 2. Difratogramas de raios - X das aluminas

Os resultados de análise de adsorção de nitrogênio estão apresentados na Tabela 2, onde se observa que os materiais possuem valores de áreas superficiais e volume de poros próximos. Entretanto se destaca a alumina ácida com maior área superficial, seguida da alumina básica e, por último, da alumina neutra.

Tabela 2. Áreas superficiais específicas e volume de poros dos materiais

\begin{tabular}{lccc}
\hline & $\begin{array}{c}\text { Alumina } \\
\text { ácida }\end{array}$ & $\begin{array}{c}\text { Alumina } \\
\text { básica }\end{array}$ & $\begin{array}{c}\text { Alumina } \\
\text { neutra }\end{array}$ \\
\hline Área superficial específica $\left(\mathrm{m}^{2} / \mathrm{g}\right)$ & 152,1 & 141,3 & 126,9 \\
Volume de poros total $\left(\mathrm{cm}^{3} / \mathrm{g}\right)$ & 0,2351 & 0,2345 & 0,2396 \\
\hline
\end{tabular}

As características ácidas e básicas dos materiais foram avaliadas através de uma titulação com $\mathrm{HCl}(0,1205 \mathrm{~mol} / \mathrm{L})$ e $\mathrm{NaOH}(0,1116$ mol/L). A alumina ácida apresentou 6,95 mmoles de $\mathrm{H}^{+} / 100 \mathrm{~g}$, a alumina básica apresentou 3,61 mmoles de $\mathrm{OH} / 100 \mathrm{~g}$, enquanto que a alumina neutra não apresentou $\mathrm{OH}^{-}$mas apresentou 3,48 mmoles de $\mathrm{H}^{+} / 100 \mathrm{~g}$.

\section{Adsorção de benzotiofeno e dibenzotiofeno}

Os resultados do processo de adsorção realizado nas amostras contendo benzotiofeno (1000 mg/L) e dibenzotiofeno (1000 mg/L) em diferentes tempos de contato estão representados nas Figuras 3 e 4 e na Tabela 3.

Observa-se que com o aumento do tempo de contato ocorre um aumento na quantidade adsorvida. O dibenzotiofeno é adsorvido em quantidade muito superior ao benzotiofeno, indicando que interage mais com a superfície. Independentemente do tipo de alumina (ácida, básica ou neutra) as quantidades adsorvidas são similares, entretanto observa-se uma tendência a maior adsorção na alumina ácida. Esta 


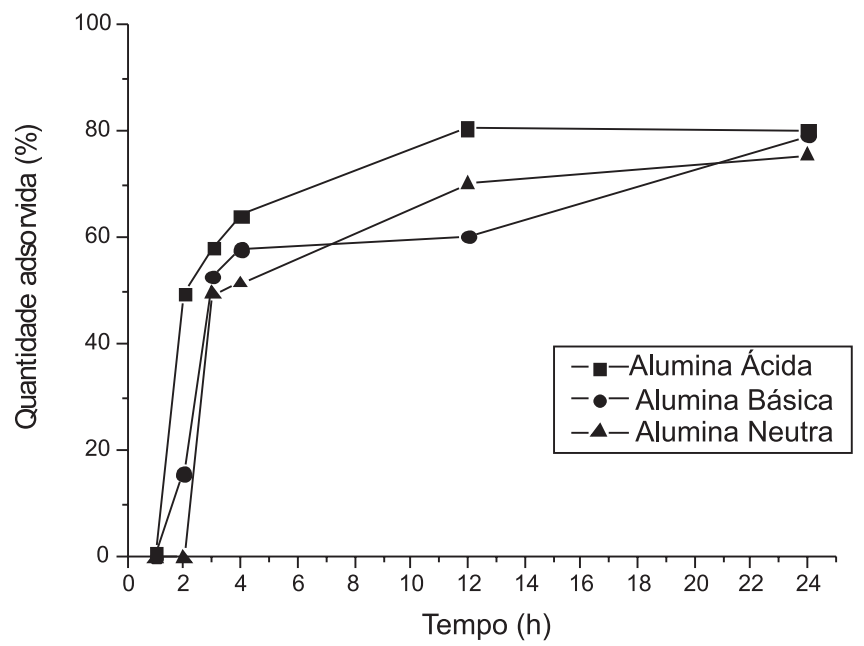

Figura 3. Quantidade adsorvida de dibenzotiofeno (\%)

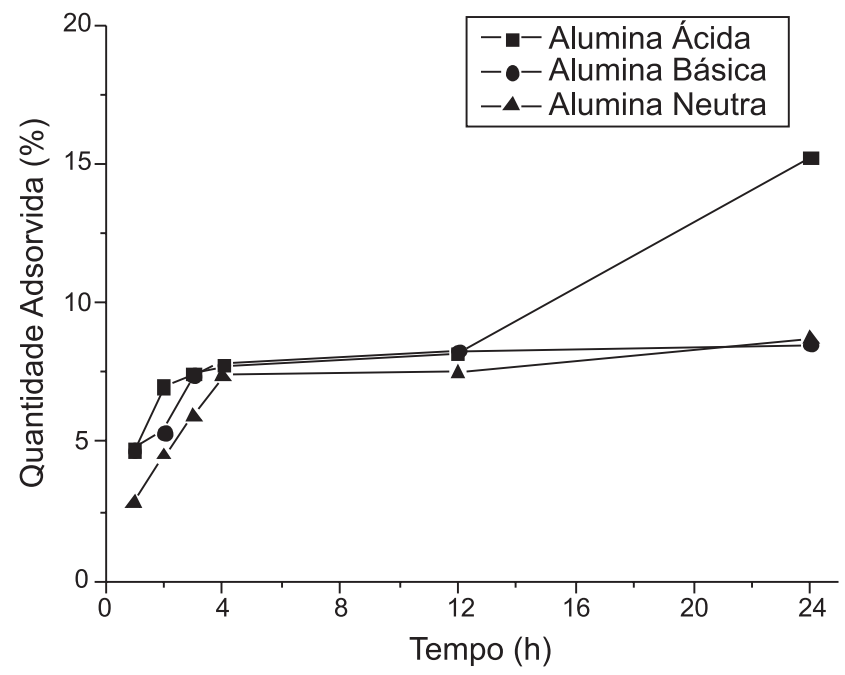

Figura 4. Quantidade adsorvida de benzotiofeno (\%)

tendência à maior adsorção na alumina ácida pode ser devida a sua maior área superficial específica e características ácidas.

A Figura 5 apresenta os resultados da análise de espectroscopia no infravermelho dos materiais antes e após o processo de adsorção com dibenzotiofeno. Observa-se o surgimento de novas bandas indicando que a adsorção ocorreu. Ao comparar com o espectro padrão do dibenzotiofeno ${ }^{13}$ observa-se que as bandas a $2900, \sim 1600$ e $\sim 1400$ $\mathrm{cm}^{-1}$ estão presentes. Entretanto não se observa a banda em $\sim 700$ $\mathrm{cm}^{-1}$ referente ao estiramento $\mathrm{C}-\mathrm{S}$, o que pode ser um indicativo de que a interação do dibenzotiofeno com a superfície da alumina seja através do enxofre como esperado.

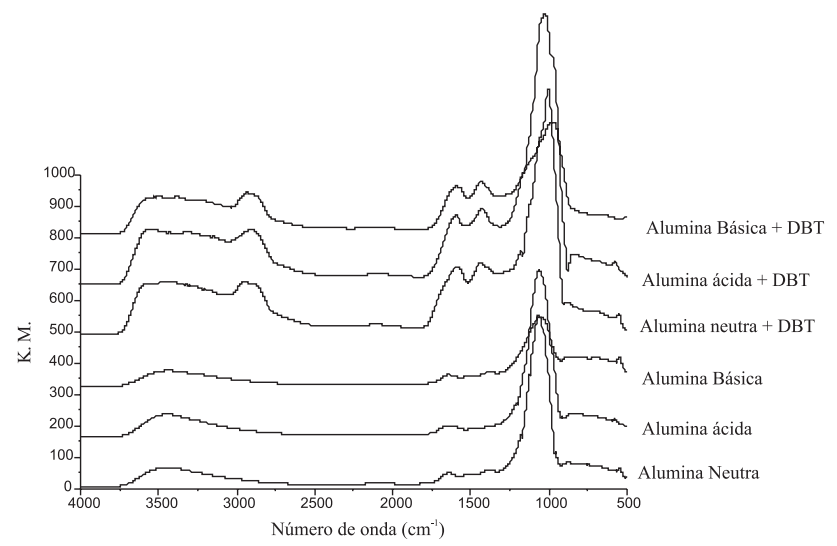

Figura 5. Espectro no infravermelho das amostras

\section{Oxidação da amostra contendo benzotiofeno e dibenzotiofeno}

O processo de oxidação foi realizado numa amostra de diesel dopado com os 2 compostos sulfurados ( $500 \mathrm{mg} / \mathrm{L}$ cada). Os cromatogramas obtidos da amostra de diesel dopado, antes e após processo de oxidação mostraram o aparecimento de dois novos picos em tempos de retenção diferente. Isto indica que os compostos sulfurados foram efetivamente oxidados não se observando a presença de benzotiofeno e dibenzotiofeno sem serem oxidados, sendo assim o processo oxidação foi totalmente efetivo.

Verifica-se que com o processo de oxidação já ocorre uma extração bastante significativa dos compostos sulfurados, chegando a $59,4 \%$ para o dibenzotiofeno oxidado e $94,7 \%$ para o benzotiofeno oxidado.

Percebe-se ainda uma diminuição dos demais picos, indicando que outros compostos de enxofre presentes na amostra de diesel comercial também são extraídos pelo processo de oxidação.

Em virtude destes resultados, para o processo de adsorção se empregou somente o dibenzotiofeno oxidado (uma nova amostra de diesel comercial dopada somente com o composto dibenzotiofeno $1000 \mathrm{mg} / \mathrm{L})$.

\section{Adsorção de dibenzotiofeno oxidado}

A critério de comparação, o mesmo processo de adsorção foi realizado em amostras oxidadas em três estágios, o composto sulfurado em estudo foi o dibenzotiofeno com um tempo de contato de $24 \mathrm{~h}$. Estes resultados são apresentados na Tabela 4 e na Figura 6; observa-

Tabela 3. Resultados da adsorção do diesel dopado com benzotiofeno e dibenzotiofeno

$\%$ Sulfurado extraído (Benzotiofeno e Dibenzotiofeno)

Tempo de

contato (h)
Alumina ácida
Alumina básica
Alumina neutra

\begin{tabular}{ccccc} 
\% benzo & \% dibenzo & \% benzo & \% dibenzo & \% benzo \\
\hline 4,7 & 0 & 4,8 & 0 & 2,8 \\
7,0 & 15,9 & 5,4 & 0 & 4,5 \\
7,5 & 52,7 & 7,4 & 49,5 & 5,9 \\
7,8 & 58,0 & 7,8 & 51,3 & 7,4 \\
8,2 & 60,3 & 8,3 & 70,2 & 7,5 \\
15,3 & 79,2 & 8,5 & 75,4 & 8,7
\end{tabular}


Tabela 4. Resultados do processo de adsorção do diesel oxidado

$\%$ Sulfurado extraído (Dibenzotiofeno oxidado - 24 h)

\begin{tabular}{lccc} 
Estágios & Alumina ácida & Alumina Básica & Alumina Neutra \\
\hline 1 & 56,0 & 54,8 & 57,9 \\
2 & 66,6 & 57,8 & 66,7 \\
3 & 69,8 & 68,6 & 67,0 \\
\hline
\end{tabular}

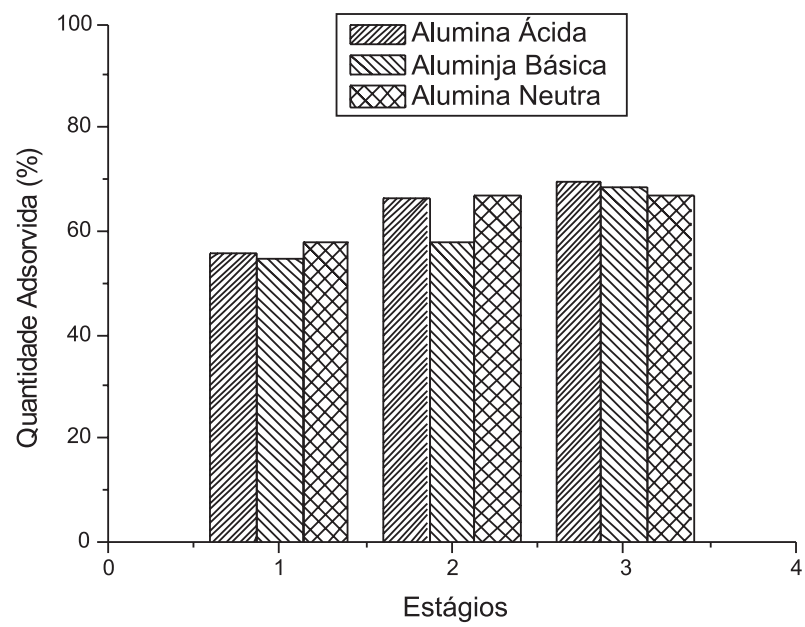

Figura 6. Quantidade adsorvida de dibenzotiofeno oxidado (\%) em diferentes estágios

\section{Estágio 1}

Al. básica
\begin{tabular}{|l|}
\hline $54,8 \%$ \\
\hline Al. ácida \\
\hline $56,0 \%$ \\
\hline Al. neutra \\
\hline $57,9 \%$ \\
\hline
\end{tabular}

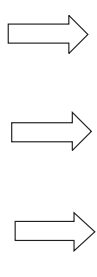

\section{Estágio 2}

Al. ácida
\begin{tabular}{|l|}
\hline $67,0 \%$ \\
\hline Al. neutra \\
\hline $66,7 \%$ \\
\hline Al. básica \\
\hline $58,2 \%$ \\
\hline
\end{tabular}

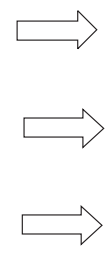

Al. neutra

$69,8 \%$

$68,6 \%$

Al. ácida

$66,6 \%$
Al. básica

Figura 7. Quantidade de dibenzotiofeno oxidado adsorvida em diferentes estágios

se que o dibenzotiofeno oxidado é adsorvido nos sólidos. O tipo de alumina não influi significativamente na capacidade de adsorção. Os níveis de remoção alcançados mesmo depois de três estágios (70\%) são inferiores ao alcançado com a adsorção nas amostras de dibenzotiofeno não oxidadas. Estes resultados indicam que para a remoção do dibenzotiofeno o processo mais adequado é a adsorção sem a oxidação, enquanto que para o benzotiofeno o processo de oxidação se mostrou mais eficiente para a remoção que o de adsorção.

Também foi realizado um experimento de adsorções em três diferentes estágios, entretanto invertendo a alumina empregada. Os resultados estão apresentados na Figura 7, onde se observa que os níveis de remoção alcançados após o terceiro estágio são similares aos dos anteriores (sem inverter a alumina), indicando mais uma vez que o tipo de alumina não influi significativamente no processo e sim o número de estágios.

\section{CONCLUSÕES}

A aplicação de aluminas como adsorventes para compostos sulfurados mostra-se como uma rota alternativa e promissora para a remoção destes compostos em combustíveis.

Os materiais adsorventes mostram uma maior seletividade na adsorção do dibenzotiofeno que o benzotiofeno.

O tempo de contato influi no processo de adsorção alcançando-se $80 \%$ de remoção para o dibenzotiofeno (não oxidado) em $24 \mathrm{~h}$.

A remoção do dibenzotiofeno é mais efetiva na amostra não oxidada, enquanto que o benzotiofeno é removido quase que totalmente no processo de oxidação.

\section{MATERIAL SUPLEMENTAR}

Os cromatogramas obtidos da amostra de diesel dopado, antes e após processo de oxidação estão representados nas Figuras $1 \mathrm{~S}$ e $2 \mathrm{~S}$, disponíveis em http://quimicanova.sbq.org.br, na forma de arquivo PDF, com acesso gratuito.

\section{REFERÊNCIAS}

1. Yang, R. T.; Hernández-Maldonado, A. J.; Yang, F. H.; Science 2003, 301, 79 .

2. Detoni, C.; Conceição, L.; Mignoni, L. M.; Pergher, S. B. C.; Resumos da 29a Reunião Anual da Sociedade Brasileira de Química, Poços de Caldas, Brasil, 2006.

3. Detoni, C.; Conceição, L.; Mignoni, L. M.; Delanora, R.; Pergher, S. B. C.; Resumos do $6^{\circ}$ Encontro Brasileiro de Adsorção, Maringá, Brasil, 2006.

4. Sepúlveda-Escribano, A.; Rodrigues-Reinoso, F.; Silvestre-Albero, J.; Rios, R. V. R. A.; Resumos do $6^{\circ}$ Encontro Brasileiro de Adsorção, Maringá, Brasil, 2006.

5. Melo, C. A.; Machado, M. C. N.; Rodrigues, D. P.; Silva, M. A.; Carvalho, M. W. N. C.; Alsina, L. S.; Barbosa, C. M. B. M.; Sousa, A. G.; Resumos do $6^{\circ}$ Encontro Brasileiro de Adsorção, Maringá, Brasil, 2006.

6. Brito, D. F.; Silva, E. F. B.; Pereira, F. A. R.; Rodrigues, D. P.; Machado, M. C. N.; Silva, M. A.; Simões, V.; Carvalho, M. W. N. C.; Barbosa, C. M. B. M.; Souza, A. G.; Resumos do $6^{\circ}$ Encontro Brasileiro de Adsorção, Maringá, Brasil, 2006.

7. Whitehurst, D. D.; Knudsen, K. G.; Wiwel, P.; Zeuthen, P.; Resumos da ACS $219^{\text {th }}$ National Meeting, San Francisco, EUA, 2000.

8. Ferraz, W.; Oliveira, L. C. A.; Dallago, R.; Conceição, L.; Catal. Commun. 2007, 8, 131.

9. Souza, W. F. de; Guimarães, J. R.; Oliveira, L. C. A.; Guerreiro, M. C.; Guarieiro, A. L. N.; Carvalho, K. T. G.; J. Mol. Catal. A: Chem. 2007, $278,145$.

10. Levy, R. E.; Rappas, A. S.; Nero, V. P.; Decanio, S. J.; Resumos do NPRA ANNUAL MEETING, New Orleans, EUA, 2001.

11. Scherer, R. P.; Pergher, S. B. C.; Souza, de W. F.; Resumos do $14^{\circ}$ Congresso Brasileiro de Catálise, Porto de Galinhas, Brasil, 2007.

12. Conceição, L.; Almeida, C. L.; Egues, S.; Dallago, R. M.; Paroul, N.; Nascimento Filho, I.; Souza, W. F.; Pergher, S. B. C.; Energy Fuels 2005, 19, 960.

13. http://spectra.galactic.com/spectraonline, acessada em Outubro 2007. 


\section{ESTUDO DE ADSORÇÃO DE COMPOSTOS SULFURADOS UTILIZANDO UM DIESEL COMERCIAL DOPADO COM BENZOTIOFENO E DIBENZOTIOFENO}

Robison P. Scherer, Álvaro L. Malvesti e Sibele B. C. Pergher*

Departamento de Química, Universidade Regional Integrada do Alto Uruguai e das Missões, Av. Sete de Setembro, 1621, 99700-000 Erechim - RS, Brasil

Wladmir Ferraz de Souza

PETROBRAS/CENPES/THPE, Ilha do Fundão, Quadra 7, 21949-900 Rio de Janeiro - RJ, Brasil

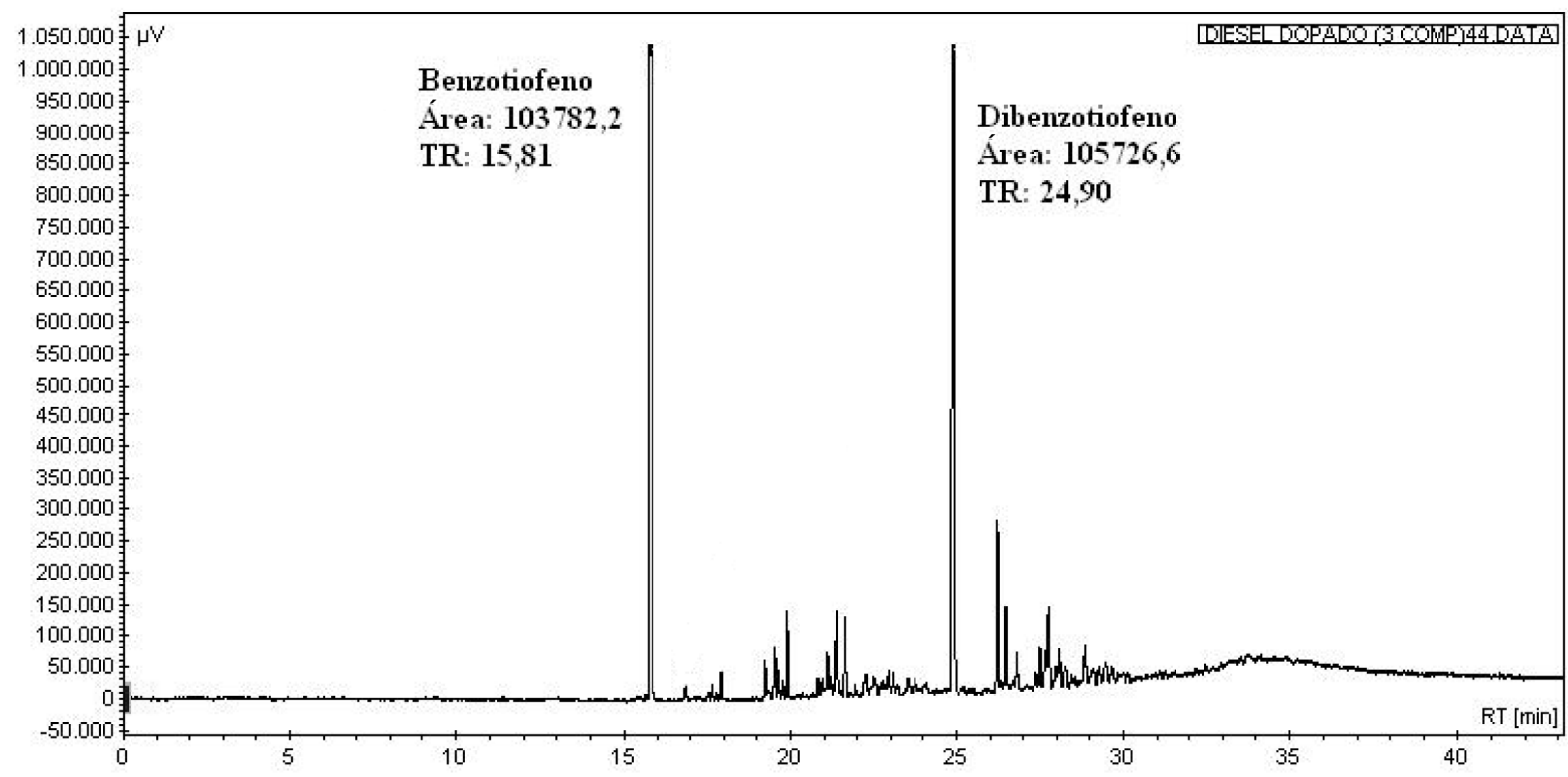

Figura 1S. Cromatograma obtido da amostra antes do processo de oxidação (analisada em GC/PFPD)

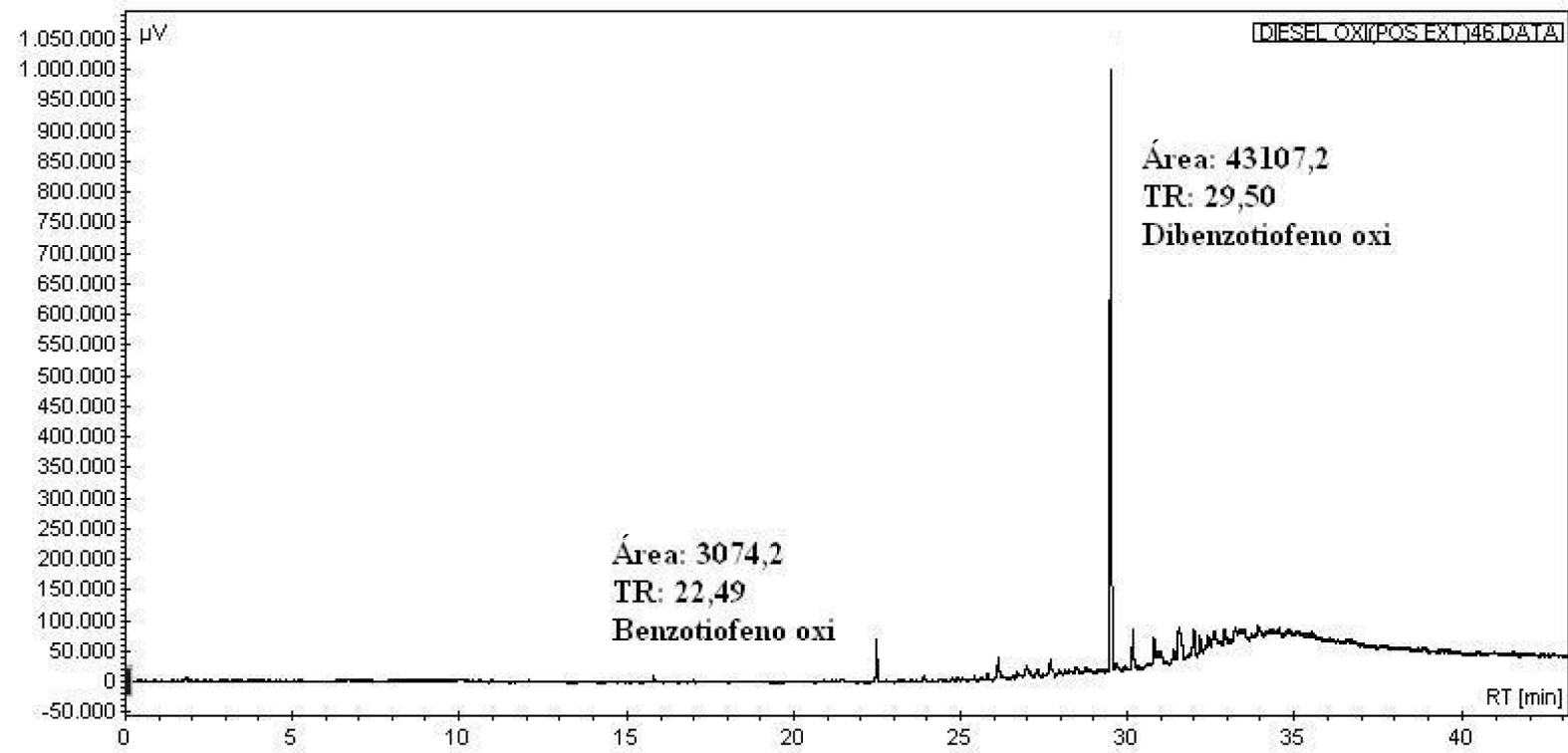

Figura 2S. Cromatograma obtido da amostra após processo de oxidação (analisada em GC/PFPD) 